\title{
Toward the Deployment of an Ultra-Wideband Localization Test Bed
}

\author{
Alexander Feldman*, Alexander Bahr ${ }^{\dagger}$, James Colli-Vignarelli ${ }^{\ddagger}$, Stephan Robert* \\ Catherine Dehollain ${ }^{\ddagger}$, and Alcherio Martinoli ${ }^{\dagger}$ \\ * Institute for Information and Communication Technologies \\ Haute Ecole d'Ingénierie et de Gestion du Canton de Vaud (HEIG-VD) \\ Email: \{alexander.feldman, stephan.robert\}@heig-vd.ch \\ $\dagger$ Distributed Intelligent Systems and Algorithms Laboratory, Ecole Polytechnique Fédérale de Lausanne (EPFL) \\ Email: \{alexander.bahr, alcherio.martionoli\}@epfl.ch \\ $\ddagger$ Radio Frequency Integrated Circuit Group, Ecole Polytechnique Fédérale de Lausanne (EPFL) \\ Email: \{james.colli-vignarelli, catherine.dehollain\}@epfl.ch
}

\begin{abstract}
The design, development, and deployment of Ultra-Wideband (UWB) localization systems involves digital and Radio-Frequency (RF) hardware, embedded software, localization algorithms, security and reliability aspects, electromagnetics, and others. Design and integration decisions affect the performance of an UWB system, in particular the most important metrics: localization accuracy and position update rate. To facilitate further development of UWB localization systems and to analyze some of the major trade-offs we share our experience in deploying the EPFL UWBLite test bed (U-Lite). We describe an approach to numerical simulation modeling that can help in the design and evaluation of UWB localization systems. To validate our approach we show experimental results with one transmitter and one receiver. Our UWB test bed includes a mobile robot platform, so we can study and evaluate the UWB performance trade-offs in realworld conditions.
\end{abstract}

\section{INTRODUCTION}

Using Ultra-Wideband (UWB) technology for localization [1] has made great advancements in recent years and commercial systems are now available [2], [3]. The localization accuracy and update rate of the existing systems however is not satisfying for many applications, particularly in adverse conditions such as Non-Line of Sight (NLoS).

In order to improve localization accuracy and update rate beyond the state-of-the-art, we developed an UWB test bed to track mobile assets. This test bed was designed and built entirely by us or our collaborators which provides us access to every component of the system. Using the mobile robot KHEPERA III [4] in combination with SwisTrack [5], a fast, accurate, and modular tracking system using overhead cameras, enables us to compare the UWB system's performance against ground truth in real time. While building up the test bed we also built models for some of the components. This enables us to simulate

The work presented in this paper was supported by the National Competence Center in Research on Mobile Information and Communication Systems (NCCR-MICS), a center supported by the Swiss National Science Foundation under grant number 5005-67322. parts of the system and quantify the amount of error that components contributes to the overall localization error of the system.

Our UWB band system uses only low-cost Commercial Off-The Shelf (COTS) components and the performance characteristics which we are trying to improve are relevant to almost all UWB applications. As a result the insights obtained from our test bed will be highly relevant for the development of future UWB localization systems.

The contributions of this paper are as follows: (1) We describe U-Lite and its major parts. (2) We show the use of a numerical model for simulating the workings of the analog transmitter and receiver front-ends. (3) We perform experimental validation and show that U-LITE can be used for localization and that the simulation results are valid. (4) We analyze some major trade-offs in the design and deployment of UWB localization systems.

At the time of writing of this paper, we have deployed a single transmitter that we can position through the mobile robot and a single receiver. While this is not sufficient for 2-D localization, we can measure the performance of the transmitter and the receiver and validate their designs. Last, although we do not quantify costs, we have achieved an economical UWB localization test bed by using readily available and cheap COTS components.

\section{Related Work}

UBISENSE [2] is a successful commercial UWB localization platform that combines Time Difference of Arrival (TDoA) and angle-of-arrival (AoA) information for increased position accuracy. To the best of our knowledge, there is no work comparing the accuracy of the UBISENSE AoA system using three receivers to a test bed that uses TDoA only and has six stationary receivers (recall that only three receivers are necessary for 2-D TDoA). While we are ultimately aiming at improving the performance of the UBISENSE state-of-the-art system, our main goal is to map the design and deployment trade-offs in building UWB localization systems. 
Our paper resembles the one of Kuhn et al. [3], we place more emphasis on practical deployment and using only Simulink ${ }^{\circledR}$ as a simulation tool. Kuhn et al. compare the position accuracy of UBISENSE to the one of the Sapphire DART system from Zebra Technologies (the latter does not use AoA) and report $\leq 10 \mathrm{~cm}$ precision for DART and $\leq 15 \mathrm{~cm}$ for UBISENSE.

A fully deployed test bed similar to ours [6] reports a maximal error of $15 \mathrm{~cm}$ by using TDoA only. To simplify the acquisition algorithms, the authors use narrow-band radios for receiver-transmitter communication. While the latter paper reports on the localization results and approaches, our paper focuses on the system design process and associated trade-offs.

We emphasize the deployment of U-LiTE as a localization test bed. UWB communication architectures are similar [7] and we will run communication experiments in the future.

\section{TEST BED}

U-LiTE consists of three or more stationary receiver assemblies and mobile transmitters mounted on top of KHEPERA III robots as shown in Fig. 1.

KHEPERA III is a small $(d=12 \mathrm{~cm})$ mobile robot [4]. Using the SwisTrack system [5] the robot's absolute position can be tracked in real time. The SwisTrack system consists of an overhead camera mounted above a $3 \mathrm{~m}$ by $3 \mathrm{~m}$ arena. The video feed from the camera is analyzed by the SwISTRACK software which extracts the position of colored LEDs attached to the modulator board on the robot to obtain an absolute position and orientation (position error: $\mu_{x}=\mu_{y}=1.2 \mathrm{~cm}, \mu_{\theta} \approx 4^{\circ}$ ). This position provides a ground truth to which the position from the UWB system can be compared.

The transmitters consist of a modulator board that generates a pulse wave and a Radio Frequency (RF) analog front-end that narrows the pulses and also provides the basic carrier frequency of $4.25 \mathrm{GHz}$. The resulting signal has a bandwidth of at least $500 \mathrm{MHz}$ [8].

The modulator board uses an embedded microcontroller (dsPIC33FJ128MC706) running at $40 \mathrm{MHz}$. By using two synchronized built-in Serial Peripheral Interface (SPI) circuits rather than directly controlling I/O-pins by microcontroller instructions, we ensure that the stability of the output pulses depends only on the crystal generating the microcontroller's clock. The modulator can generate arbitrary UWB pulse sequences up to 8000 symbols long with a Pulse Repetition Rate (PRR) of up to $10 \mathrm{MHz}$. By using data modulation techniques such as On-Off keying or Pulse Position Modulation (PPM), we can transmit symbol sequences that can be used for robot identification, security, or other purposes. The generated pulses are $25 \mathrm{~ns}$ long and are shortened to 8 ns by discrete logic circuitry before being fed into the RF front-end. In addition to generating the pulse sequences the modulator also generates the various required supply voltages from the robot's

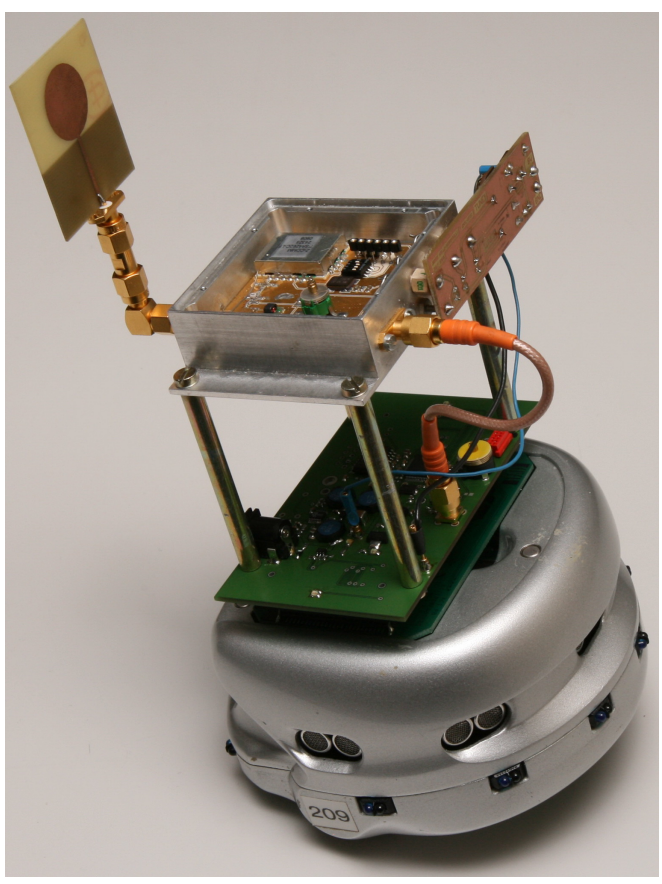

Fig. 1. The mobile transmitter consisting of a KHEPERA III robot, the modulator (green circuit board), the UWB transmitter and the antenna

battery.

The transmitter's RF stage is depicted in the left part of Fig. 2. An integrated PLL sine wave oscillator running at $4.25 \mathrm{GHz}$ is connected to the antenna output through a mixer. The mixer behaves like a switch when driven by a square digital signal. By using this part of the RF frontend, the pulses generated by the modulator are further shortened to $3 \mathrm{~ns}$. The exact duration of the pulse can be finely tuned with a trimmer. The transmitter is described in detail in [8].

The design of the transmitter and receiver antennae is the same and is described in [9]. We have performed the experiments in this paper using the traditional monopole omni-directional antenna that is visible in Fig. 1 and an improved antenna is currently in a prototype phase.

The receiver RF stage is a direct-conversion circuit and is shown in the right side of the diagram in Fig. 2. The signal from the antenna is amplified and band-pass filtered by a cascade of two Low-Noise Amplifiers (LNAs) with voltage controlled gain [10] and then down-converted with an I/Q mixer driven by a $4.25 \mathrm{GHz}$ sine wave. The I/Q mixer is used to remove any beat that could occur due to frequency mismatch between the transmitter and receiver local oscillators.

The down-converted and amplified signal is fed to a dual 1.5 GS/s ADC08D1500 ADC on the receiver sampling board [11]. The sampling frequency can be configured in the range from $2.816 \mathrm{GHz}$ to $2.904 \mathrm{GHz}$, in $8 \mathrm{MHz}$ increments. The resolution of the ADC is 8 bits and the input range is $650 \mathrm{mV}$. The fast ADC generates $2.824 \mathrm{GBps}$ at its default sampling rate, and, for such large throughput 


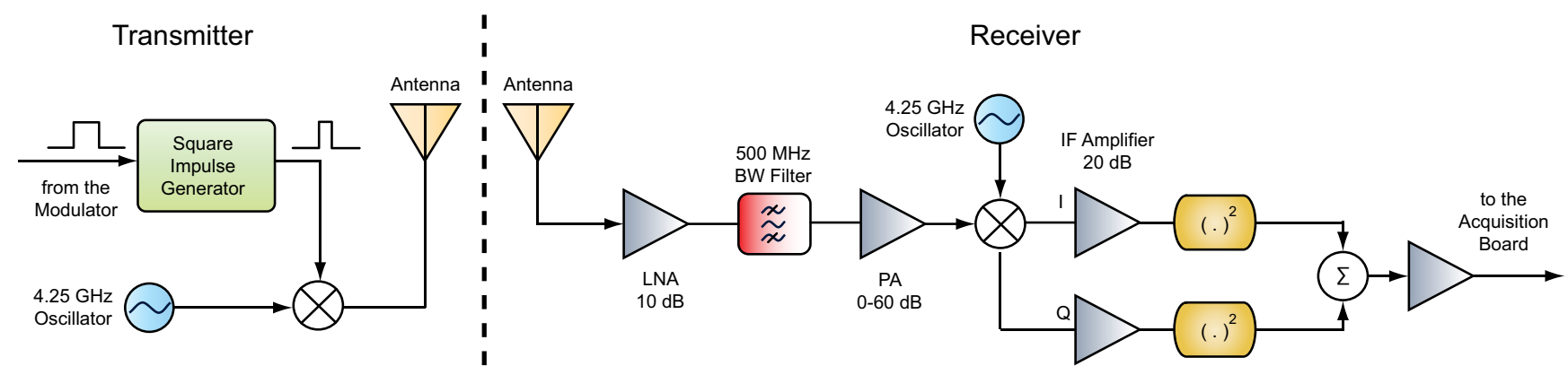

Fig. 2. Overview of the RF analog parts of the transmitter and the receiver

of data, there is not much choice but to deserialize with a Field-Programmable Gate Array (FPGA).

The receiver sampling board uses an Altera Cyclone II EP2C70 with 68416 logic elements and $140 \mathrm{~Kb}$ of RAM. The architecture uses a data-driven main clock, i.e., the FPGA is clocked by the ADC. The ADCs in the receiver sampling boards are driven by LMX2326 PhasedLock Loops (PLLs) that are phase-synchronized with an external $40 \mathrm{MHz}$ laboratory sine generator. The FPGA communicates with the localization base-station (a PC) by using an FT245 serial-to-USB converter.

The receiver FPGA devices are flashed with the Basic Impulse Radio Data acquisition System (BIRDS). BIRDS implements custom IP modules for interfacing with the ADC, the PLL, and the serial-to-USB converter. BIRDS also has the NIOS II embedded processor by Altera for faster implementation of signal processing, acquisition, and localization algorithms as well as for debugging and troubleshooting.

BIRDS provides the basic functionality of a Digital Storage Oscilloscope (DSO) and computes the position of pulses by detecting thresholds or by using a matched filter. The latter cannot be done in real-time due to the relatively low-end FPGA chip. Interestingly, a comparison of pulse detection algorithms [12] run under realistic simulations concludes that threshold (edge) detection results in higher position accuracy compared to, for example, matched filter. The authors attribute that to phase noise of the clocks and sampling jitter. The pulse position information can be sent directly to the localization base-station for computing the robot position by using multilateration.

\section{Numerical Model}

When modifying the hardware and evolving the software of U-LiTE we use a high-fidelity model, where possible from first principles, to asses the design trade-offs and to estimate the test bed performance. In this section we present a model of the RF subsystem shown in Fig. 2. The use of this model should motivate the use of I/Q demodulation in the receiver front-end (recall that the transmitter does not use I/Q modulation and the demodulation is only to counter the "beat" effect from slight differences in the transmitter and receiver frequencies).
COTS oscillators such as the ones used in our UWB transmitter and receiver produce slightly different waveforms due to manufacturing tolerances and environmental factors. As we will see in this section, such differences negatively affect the received signal, increase the Signalto-Noise Ratio (SNR), and subsequently increase the Bit Error Rate (BER) in communication applications or decrease the accuracy in localization applications.

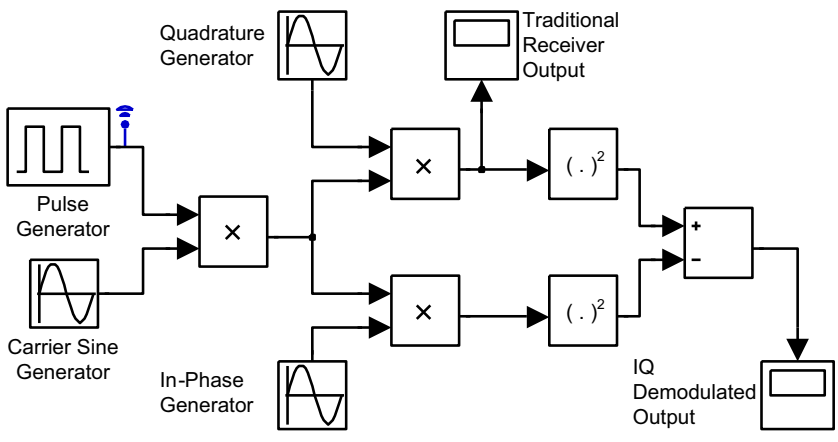

Fig. 3. A Simulink ${ }^{\circledR}$ UWB communication model of the RF analog front-end

We have used numerical simulation to compare the performance of a traditional receiver to that of one using I/Q demodulation. The Simulink ${ }^{\circledR}$ model is shown in Fig. 3. The input signal is a pulse wave with a period of 1 us and a duty cycle of $1 \%$. It is mixed with a sine wave with a nominal frequency of $4.25 \mathrm{GHz}$. On the receiver side, the signal is down-converted by using $4.20 \mathrm{GHz}$ sine and cosine waves (the difference in the oscillator frequencies is exaggerated in order to make the example clear). The resulting in-phase and quadrature parts of the signal are then squared and summed to produce the final received signal. The output of the quadrature generator is tapped to simulate the work of a traditional receiver.

Figure 4(a) shows the simulated output signal of a traditional receiver (one that does not use I/Q demodulation) while Fig. 4(b) shows the simulated output signal of the improved receiver design that uses I/Q demodulation. Figure 5 shows the first $250 \mathrm{~ns}$ of the traditional demodulated signal shown in Fig. 4(a). Clearly, it is easier to compute the positions of the pulses in the signal received by the I/Q demodulator. 


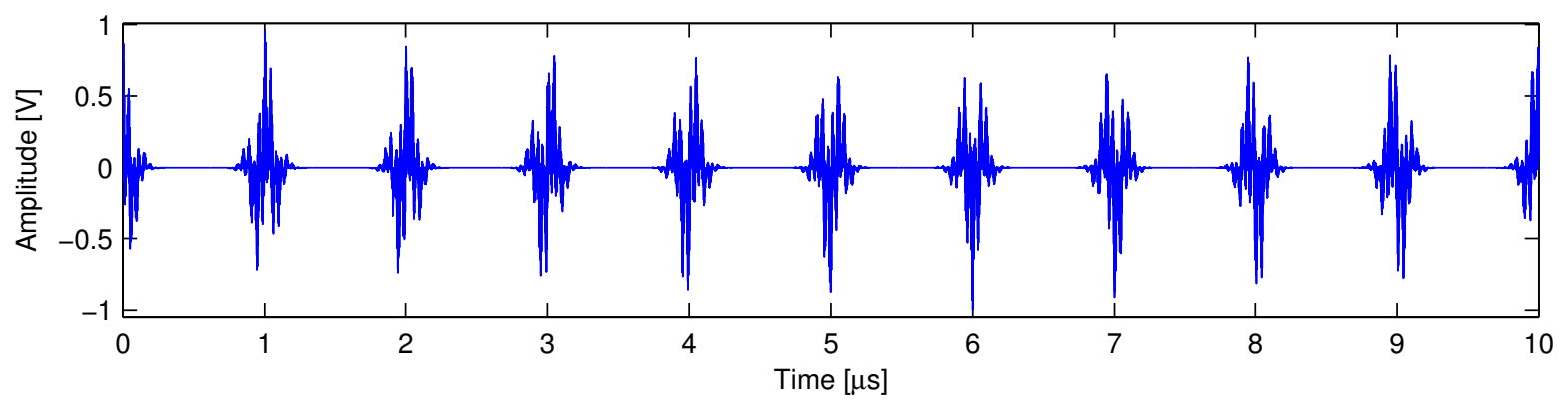

(a) Traditional receiver

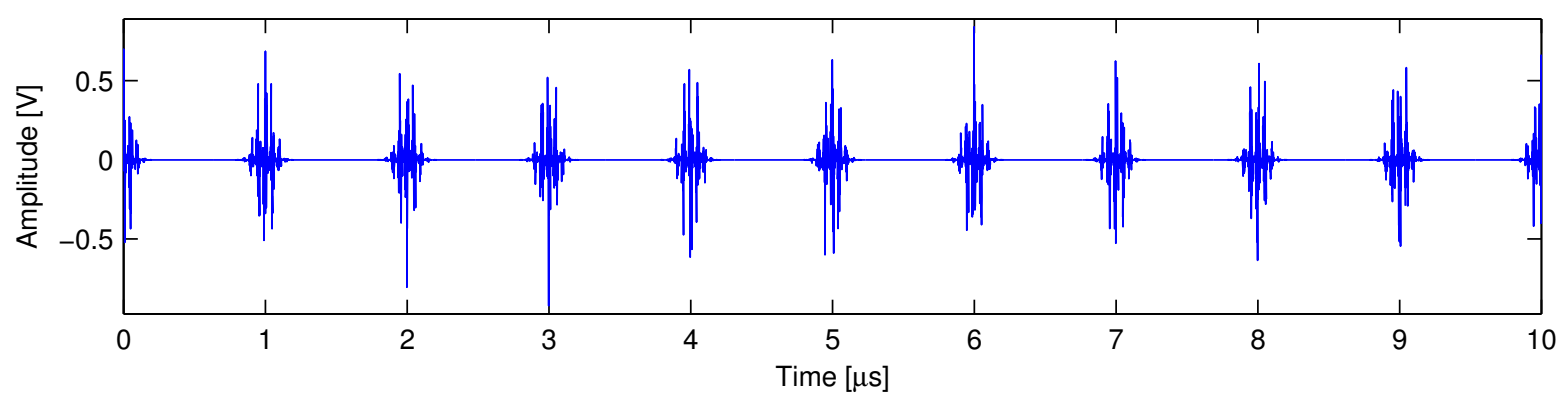

(b) Receiver with $\mathrm{I} / \mathrm{Q}$ demodulation

Fig. 4. Simulated received signal with traditional and proposed receivers

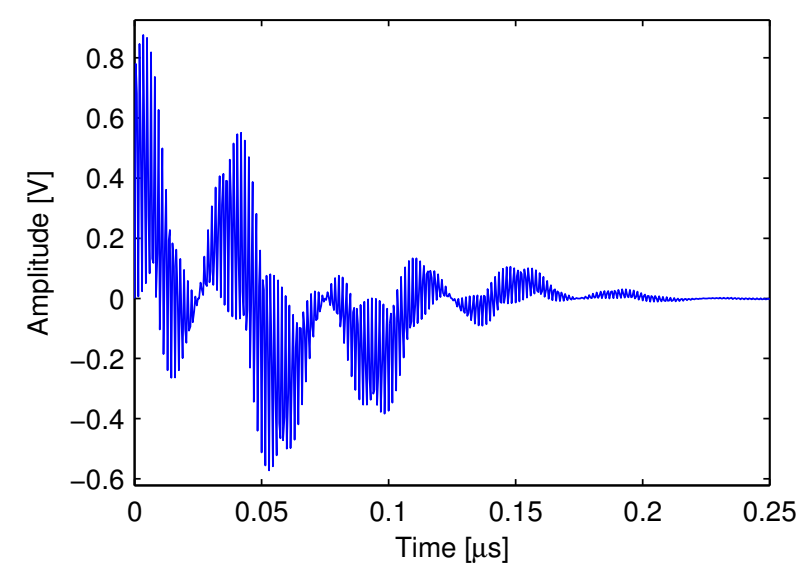

Fig. 5. The first $250 \mathrm{~ns}$ of the signal shown in Fig. 4(a)

We have implemented threshold (leading edge) pulse detection. A pulse is detected when $V>0.6[\mathrm{~V}]$ after which the detection is paused for 0.5 us. After detecting the positions of the 10 pulses this way we have computed the Mean Square Error (MSE) from the transmission times. The traditional receiver has a MSE of $1.94 \mathrm{~ns}$. The receiver that uses I/Q demodulation has a MSE of $0.5 \mathrm{~ns}$. The $\mathrm{I} / \mathrm{Q}$ demodulated design we propose gives us a significant improvement of $1.44 \mathrm{~ns}$ that translates to $0.43 \mathrm{~m}$ (the TDoA localization MSE is going to be smaller due to the averaging of several samples). Further, the accuracy values are much better in practice as differences in the oscillator frequencies are much less dramatic than the ones assumed in this simulation.

\section{Experimental Validation}

Figure 6 shows 0.18 us of the received real signal as sampled by the ADC. The second pulse in Fig. 6 is sent with a 100 ns delay after the first one $(10 \mathrm{MHz}$ PRR see Sec. III). We see that there is little noise and despite the fact that the signal is captured in a lab and not in an anechoic chamber, there are no significant reflections or signal distortions.

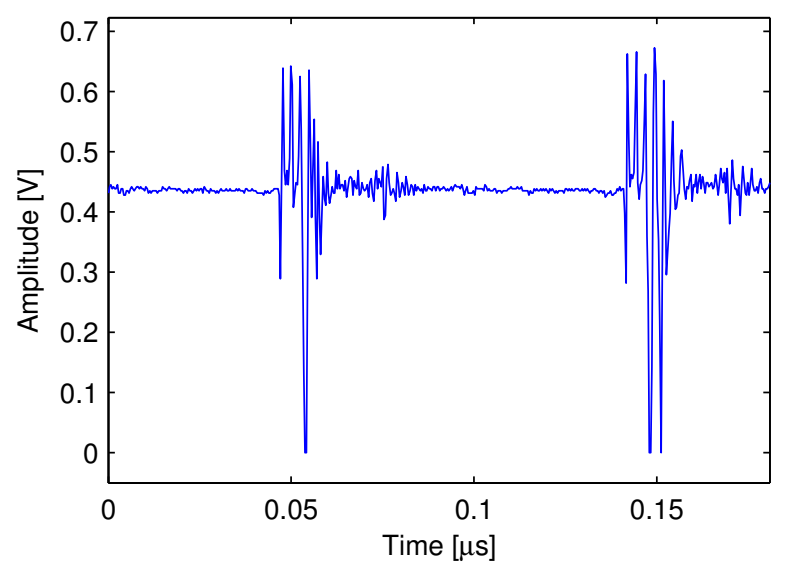

Fig. 6. Two consecutive pulses as captured by the ADC

Next we compute the clock differences between the arriving pulses by using threshold detection, similar to the one described in Sec. IV. For each of the configurable sampling frequency, we have captured 163840 pulses in 10 consecutive sessions. 


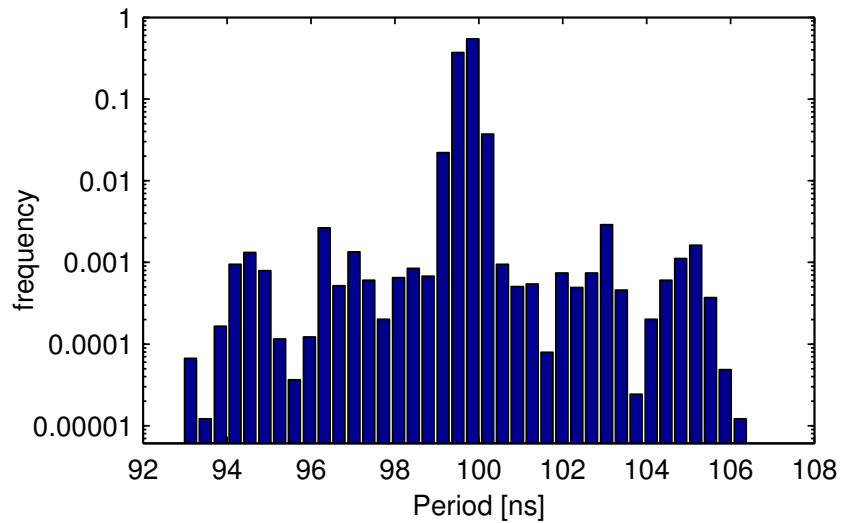

Fig. 7. Distribution of the inter-pulse periods of the received waveform

The histogram in Fig. 7 (notice the logarithmic Yaxis) shows the distribution of the differences of the pulse positions at the default $2.824 \mathrm{GHz}$ sampling frequency. The bins in this histogram are $0.35 \mathrm{~ns}$ wide. The four central bins contain $97.6 \%$ of the received pulses, i.e., $97.6 \%$ of the received pulses arrive within $1.4 \mathrm{~ns}$ of their scheduled arrival time. When plotting the histogram shown in Fig. 7, we have discarded 316 out of 163840 data points $(2.4 \%)$ that would lie in the tails of the distribution, i.e., pulses that are detected 6.9 ns earlier or 6.2 ns later than their scheduled arrival time. These outliers are due to undetected pulses, and we hypothesize that the reason for that is the lack of automated gain control at this stage of the test bed deployment.

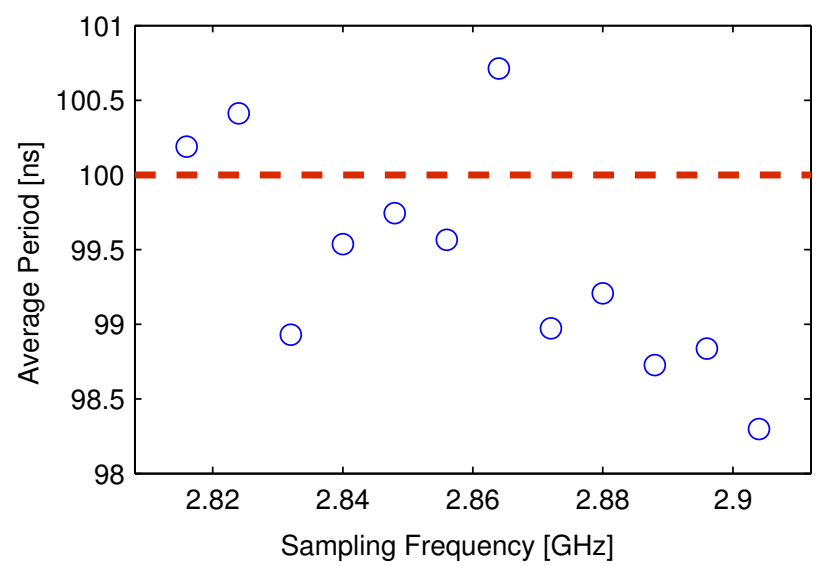

Fig. 8. Average measured pulse arrival period for different sampling frequencies

Figure 8 shows the mean arrival period of the I/Q demodulated receiver for different sampling frequencies. We see that the results are closest to the nominal $100 \mathrm{~ns}$ for the lowest rate of $2.816 \mathrm{GHz}$ and the error increases for higher frequencies. Part of the error is contributed by the ADC which under-performs at higher sampling rates. In all the cases, however, the error is a few nanoseconds, which, given the small sample size, indicates a good receiver performance for localization and ranging applications.

\section{Vi. Conclusions and Future Work}

Designing and deploying UWB localization systems is a multidisciplinary topic with each step potentially affecting the final performance of the system. In this paper we have discussed the design and deployment of U-LiTE. We show how to use basic numerical modeling to motivate the use of I/Q demodulation-based UWB receiver frontend in place of a traditional one. Experiments performed with our single-transmitter single-receiver setup indicate good detection accuracy of the pulse positions.

Our immediate goal is to fully deploy and integrate the U-LiTE test bed (recall that at the time of writing of this paper we have experimented with one transmitter and one receiver only).

We consider dense NLoS environments as the biggest challenge to UWB localization. We plan to improve our channel model to accurately simulate such environments. This will allow us to propose new signal acquisition algorithms, improve methods for data fusion on the robotic side and, eventually, improve the UWB localization performance in NLoS.

\section{REFERENCES}

[1] S. Gezici, Z. Tian, G. B. Giannakis, H. Kobayashi, A. F. Molisch, H. V. Poor, , and Z. Sahinoglu, "Localization via ultra-wideband radios: A look at positioning aspects for future sensor networks," IEEE Signal Processing Magazine, vol. 22, no. 4, pp. 70-84, 2005

[2] P. Steggles and S. Gschwind, "The ubisense smart space platform," in Proc. PERVASIVE'05, 2005.

[3] M. J. Kuhn, C. Zhang, S. Lin, M. R. Mahfouz, and A. E. Fathy, "A system level design approach to UWB localization," in Proc. MTT'09, 2009, pp. 1409-1412.

[4] A. Prorok, A. Arfire, A. Bahr, J. R. Farserotu, and A. Martinoli, "Indoor navigation research with the Khepera III mobile robot: An experimental baseline with a case-study on ultra-wideband positioning," in Proc. IPIN'10, 2010.

[5] T. Lochmatter, P. Roduit, C. Cianci, N. Correll, J. Jacot, and A. Martinoli, "SwisTrack - A Flexible Open Source Tracking Software for Multi-Agent Systems," in Proc. IROS'08, 2008, pp. 4004-4010.

[6] S. Krishnan, P. Sharma, Z. Guoping, and O. H. Woon, "A UWB based localization system for indoor robot navigation," in Proc. ICUWB'07, 2007, pp. 77-82.

[7] D. D. Wentzloff, R. Blázquez, F. S. Lee, B. P. Ginsburg, J. Powell, and A. P. Chandrakasan, "System design considerations for ultra-wideband communication," IEEE Communications Magazine, vol. 43, no. 8, pp. 114-121, 2005.

[8] J. Colli-Vignarelli and C. Dehollain, "A discrete-components impulse-radio ultra-wide band (IR-UWB) transmitter," IEEE Trans. on Microwave Theory and Techniques, 2011.

[9] G. Q. D. de Leon, J.-F. Zürcher, and A. Skrivervik, "Omnidirectional pulse dispersion of planar circular monopoles," in Proc. UWB'09, 2009, pp. 395- 399.

[10] J. Colli-Vignarelli and C. Dehollain, "A discrete-components impulse-radio UWB Low-Noise Amplifier with voltage controlled-gain," in Proc. PRIME'10, 2010.

[11] P. Tomé, C. Robert, R. Merz, C. Botteron, A. Blatter, and P.A. Farine, "UWB-based local positioning system: From a smallscale experimental platform to a large-scale deployable system," in Proc. IPIN'10, 2010.

[12] M. J. Kuhn, C. Zhang, B. Merkl, D. Yang, Y. Wang, M. R. Mahfouz, and A. E. Fathy, "High accuracy UWB localization in dense indoor environments," in Proc. ICUWB'08, vol. 2, 2008, pp. $129-132$. 